\title{
A CLINICAL STUDY OF ANTERIOR UVEITIS
}

\author{
Proddatoori Kishore Kumar' ${ }^{1}$ Puduru Sanjeeva Kumar², Pyda Sasi ${ }^{3}$, Modugula Ramesh Chandra ${ }^{4}$
}

${ }^{1}$ Assistant Professor, Department of Ophthalmology, Santhiram Medical College and General Hospital, Nandyal. ${ }^{2}$ Associate Professor, Department of Ophthalmology, Santhiram Medical College and General Hospital, Nandyal. ${ }^{3}$ Senior Resident, Department of Ophthalmology, Santhiram Medical College and General Hospital, Nandyal. ${ }_{4}^{4}$ Associate Professor, Department of Ophthalmology, Santhiram Medical College and General Hospital, Nandyal.

\section{ABSTRACT}

\section{BACKGROUND}

Uveitis, a complex intraocular inflammatory disease results from several aetiological entities. The cause of inflammation might be infectious agent or trauma, but in most cases the underlying mechanism appears to be autoimmune in nature.

Objective of this study is to evaluate the aetiological pattern, treatment and its outcome and complications of anterior uveitis.

\section{MATERIALS AND METHODS}

A prospective clinical study was done in the Department of Ophthalmology, Santhiram Medical College, Nandyal during December 2014 to May 2016. All patients between 20 - 80 yrs. of age clinically presenting with anterior uveitis were studied. A thorough clinical evaluation followed by investigations was done to determine the aetiology. Patients were put on specific and non-specific treatment and were followed up for a period of 6 months. Complications were noted.

\section{RESULTS}

The aetiology of uveitis remained unknown in most cases (42\%). Most common cause was observed to be blunt trauma (20\%) followed by phacolytic (12\%). Most cases responded well to treatment. Commonest complication was persistent posterior synechiae $(23.64 \%)$ and cataract was the second common $(14.54 \%)$.

\section{CONCLUSION}

Aetiological diagnosis remains undetermined in majority of cases. A thorough examination and investigation is required in each case to facilitate a final diagnosis. Prompt treatment ensures good visual outcome. Ocular morbidity is common in chronic and recurrent cases.

\section{KEYWORDS}

Anterior uveitis, Aetiology, Treatment Complication.

HOW TO CITE THIS ARTICLE: Kumar PK, Kumar PS, Sasi P, et al. A clinical study of anterior uveitis. J. Evolution Med. Dent. Sci. 2017;6(23):1939-1946, DOI: 10.14260/Jemds/2017/425

\section{BACKGROUND}

Uveitis is one of the most common forms of intraocular inflammation and affects mainly children and young adults. It includes a large group of intraocular inflammatory diseases of diverse aetiology. ${ }^{1}$ The precise cause of anterior uveitis is often obscure and the correct diagnosis is often challenging. The cause of inflammation might be infectious agent or trauma, but in most cases the underlying mechanism appears to be autoimmune in nature. ${ }^{2}$ To enhance understanding and management of ocular inflammation, International Ocular Inflammation Society (IOIS) has been founded. ${ }^{3}$

On several occasions, it reflects diseases that are developing elsewhere in the body and uveitis may be the first evidence of such systemic diseases. ${ }^{1}$ Variation in the spectrum of disease is largely due to complex geographic, ecological, racial, nutritional and socioeconomic differences.

Financial or Other, Competing Interest: None

Submission 01-02-2017, Peer Review 04-03-2017,

Acceptance 11-03-2017, Published 20-03-2017.

Corresponding Author:

Dr. Proddatoori Kishore Kumar,

Assistant Professor,

Department of Ophthalmology,

Santhiram Medical College and General Hospital,

Nandyal.

E-mail:drkishor.arien@gmail.com

DOI: $10.14260 /$ jemds $/ 2017 / 425$

\section{(c) $($ ) $\odot$}

The anterior uveitis is the most common type of all uveitic entities (57.4\%). Based on overall clinical presentation, acute unilateral, non-infectious and nongranulomatous forms occur more frequently. Idiopathic anterior uveitis is more common in all age groups. Mean age at presentation is 38.3 years and commonly affects middle aged (17 - 59 years). It is more common in males $(61.3 \%)$ compared to females (38.6\%). 4

Anterior uveitis is most common form of uveitis and accounts for an annual incidence rate of about 17 cases per $1,00,000$ populations. $^{5}$

\section{Aims and Objectives}

To study-

- Clinical presentation.

- Complications.

- Investigations to establish the exact cause and accurate diagnosis.

- Response to treatment and prognosis in terms of visual outcome.

\section{MATERIALS AND METHODS}

A prospective clinical study was conducted. The material for this study included, 50 patients between age 20 and 80 years, attending outpatient department, Department of Ophthalmology, Santhiram Medical College, Nandyal between 
December 2014 to May 2016 with signs and symptoms of anterior uveitis.

The anterior uveitis following penetrating ocular injuries, corneal ulcer, intraocular surgeries and if associated with intermediate, posterior or panuveitis were excluded from this study. Masquerade syndromes presenting as anterior uveitis has also been excluded.

A standard clinical proforma was filled in all cases, which included salient feature in history, visual acuity using Snellen's visual acuity chart, clinical findings, laboratory investigations and the final aetiology. All patients were examined under slit lamp.

Details on disease severity, laterality, chronicity, ocular signs and associated systemic conditions were noted.

Presentation was considered as unilateral if active inflammation was present in only one eye and bilateral if both eyes presented with active inflammation.

Intraocular inflammation was assigned anterior uveitis based on International Uveitis Study Group Criteria.

The inflammation was defined as acute if symptoms were present for less than three months, chronic if symptoms were present for three months or more and recurrent if two or more episodes of inflammation separated by a disease free period.

Anterior uveitis was defined granulomatous if large keratic precipitates, nodules at pupillary margin (Koeppe nodules) or nodules on or within the anterior iris stroma (Busacca nodules) were present.

A short differential diagnosis was made in each case. Subsequently, a tailored laboratory investigation was carried out

\section{Laboratory Investigations}

- Complete blood counts,

- Erythrocyte sedimentation rate,

- Urine and Stool examination,

- Mantoux test,

- Serological tests for Syphilis, HIV, HbsAg, Rheumatoid factor, HLA B-27, Serum ACE was done in all cases.

\section{Radiological Investigations}

X-ray of chest, lumbosacral and knee joints to rule out sarcoidosis, tuberculosis and other connective tissue disorders. Other special investigations were considered whenever necessary.

Consultation was done with other medical specialties whenever needed.

Final aetiological diagnosis was made based on history, clinical features, laboratory investigations and systemic evaluation by other medical specialties.

The anterior uveitis was considered to have idiopathic aetiology when it was not associated with HLA-B27 haplotype and neither with defined clinical syndromes nor with definitive aetiology.

All patients were treated medically with topical steroids (prednisolone acetate 1\%) and topical cycloplegic mydriatics (atropine or homatropine). Steroids frequency was titrated according to severity of uveitis.

Appropriate treatment was given whenever aetiology was known.
Systemic antimicrobials were administered when infectious agent was found to be the cause.

Systemic steroids were used when inflammation was severe, not responding to treatment and patients with macular oedema.

Cases of anterior uveitis with secondary glaucoma were treated with T. Acetazolamide $250 \mathrm{mg}$ BD/TID and/or Timolol 0.5\% eye/drops BD along with topical steroids. Each patient was followed up for 6 months. The complications were noted and the response to treatment was recorded and evaluated in each patient.

\section{RESULTS}

The present study was conducted in the Department of Ophthalmology, Santhiram Medical College, Nandyal during December 2014 to May 2016; 50 patients in the age group of 20 - 80 yrs. were studied and during the study following observations were made.

\begin{tabular}{|c|c|c|c|}
\hline Sl. No. & Age (yrs.) & Number & Percentage \\
\hline 1 & $20-30$ & 20 & 40 \\
\hline 2 & $31-40$ & 12 & 24 \\
\hline 3 & $41-50$ & 8 & 16 \\
\hline 4 & $51-60$ & 5 & 10 \\
\hline 5 & $61-70$ & 3 & 6 \\
\hline 6 & $71-80$ & 2 & 4 \\
\hline \multicolumn{4}{|c|}{ Table 1. Age Distribution } \\
\hline
\end{tabular}

Most cases were observed between the age group 20 - 40 years. Less common in above 60 years.

\begin{tabular}{|c|c|c|c|}
\hline Sl. No. & Sex & Number & Percentage \\
\hline 1 & Male & 28 & 56 \\
\hline 2 & Female & 22 & 44 \\
\hline \multicolumn{4}{|c|}{ Table 2. Sex Distribution } \\
\hline
\end{tabular}

\begin{tabular}{|c|c|c|c|}
\hline Sl. No. & Age (yrs) & Number & Percentage \\
\hline 1 & Unilateral & 45 & 90 \\
\hline 2 & Bilateral & 5 & 10 \\
\hline \multicolumn{2}{|c|}{ Total } & 50 & 100 \\
\hline
\end{tabular}

\begin{tabular}{|c|c|c|c|}
\hline Sl. No. & Occupation & Number & Percentage \\
\hline 1 & Labourer & 24 & 48 \\
\hline 2 & Officials & 11 & 22 \\
\hline 3 & Housewives & 10 & 20 \\
\hline 4 & Business & 3 & 6 \\
\hline 5 & Student & 2 & 4 \\
\hline \multicolumn{4}{|c|}{ Table 4. Occupation } \\
\hline
\end{tabular}

In the present study incidence of anterior uveitis was highest amongst the labourers (48\%) followed by officials (22\%), then housewives $(20 \%)$ and less common among businessman (6\%) and students (4\%).

\begin{tabular}{|c|c|c|c|}
\hline Sl. No. & Presentation & Number & Percentage \\
\hline 1 & Acute & 38 & 76 \\
\hline 2 & Chronic & 9 & 18 \\
\hline 3 & Recurrent & 3 & 6 \\
\hline \multicolumn{2}{|c|}{ Total } & 50 & 100 \\
\hline
\end{tabular}


In the present study, it was observed that most common presentation was acute anterior uveitis, accounting for $76 \%$, then chronic $18 \%$ and only $6 \%$ of the patients had recurrent anterior uveitis.

\begin{tabular}{|c|c|c|c|}
\hline Sl. No. & Type & Number & Percentage \\
\hline 1 & Non-granulomatous & 45 & 90 \\
\hline 2 & Granulomatous & 5 & 10 \\
\hline & Total & 50 & 100 \\
\hline
\end{tabular}

\begin{tabular}{|c|c|c|c|}
\hline Sl. No. & Aetiology & Number & Percentage \\
\hline 1 & Idiopathic & 21 & 42 \\
\hline 2 & Blunt trauma & 10 & 20 \\
\hline 3 & Phacolytic & 6 & 12 \\
\hline 4 & Herpes zoster & 5 & 10 \\
\hline 5 & Tuberculosis & 3 & 6 \\
\hline 6 & Septic focus & 1 & 2 \\
\hline 7 & $\begin{array}{c}\text { Iridocyclitis associated } \\
\text { with arthritis }\end{array}$ & 1 & 2 \\
\hline 8 & $\begin{array}{c}\text { Fuchs' heterochromic } \\
\text { iridocyclitis }\end{array}$ & 1 & 2 \\
\hline 9 & Leprosy & 1 & 2 \\
\hline 10 & $\begin{array}{c}\text { Inflammatory bowel } \\
\text { disease }\end{array}$ & 1 & 2 \\
\hline \multicolumn{3}{|c|}{ Table 7. Aetiological Distribution } \\
\hline
\end{tabular}

In this study, aetiology remain undetermined in 21 (42\%) cases and specific diagnosis was reached in 29 (58\%) cases. Anterior uveitis following blunt trauma was seen in 10 cases (20\%) and phacolytic uveitis was detected in 6 cases $(12 \%)$. Herpes zoster was responsible in $5(10 \%)$ cases and tuberculosis in $3(6 \%)$ cases. Iridocyclitis associated with arthritis, septic focus, Fuchs' heterochromic iridocyclitis, leprosy and inflammatory bowel disease was observed in 1 case (2\%) each.

\begin{tabular}{|c|c|c|c|c|c|}
\hline \multirow{2}{*}{$\begin{array}{c}\text { Sl. } \\
\text { No. }\end{array}$} & \multirow{2}{*}{$\begin{array}{c}\text { Visual } \\
\text { Acuity }\end{array}$} & $\begin{array}{c}\text { Before Treatment } \\
\text { Eo. of } \\
\text { Eyes }\end{array}$ & Percentage & $\begin{array}{c}\text { No. of } \\
\text { Eyes }\end{array}$ & Percentage \\
\hline 1 & PL + PR + & 4 & 7.27 & - & - \\
\hline 2 & $<6 / 60$ & 6 & 10.91 & - & - \\
\hline 3 & $6 / 60$ & 9 & 16.36 & 1 & 1.82 \\
\hline 4 & $6 / 36$ & 5 & 9.09 & 3 & 5.45 \\
\hline 5 & $6 / 24$ & 6 & 10.91 & 2 & 3.64 \\
\hline 6 & $6 / 18$ & 7 & 12.73 & 4 & 7.27 \\
\hline 7 & $6 / 12$ & 11 & 20 & 6 & 10.91 \\
\hline 8 & $6 / 9$ & 6 & 10.91 & 14 & 25.46 \\
\hline 9 & $6 / 6$ & 1 & 1.82 & 25 & 45.45 \\
\hline \multicolumn{7}{|c|}{ Table 8. Visual Acuity before and after Treatment } \\
\hline
\end{tabular}

The above table shows the visual acuity observed in 55 eyes before and after treatment. Before treatment 4 eyes had visual acuity PL+PR+ (7.27\%), 6 eyes had less than $6 / 60$ (10.91\%), 9 eyes $6 / 60$ (16.36\%), 5 eyes $6 / 36$ (9.09\%), 6 eyes $6 / 24(10.91 \%), 7$ eyes 6/18 (12.73\%), 11 eyes $6 / 12$ (20\%), 6 eyes $6 / 9(10.91 \%)$ and 1 eye $6 / 6$ (1.82\%). Following treatment, $70.91 \%$ of patients regained visual acuity of $6 / 9$ or better. In a few patients, visual acuity improved only marginally because of associated complications, such as complicated cataract and secondary glaucoma commonly seen in chronic and recurrent cases.

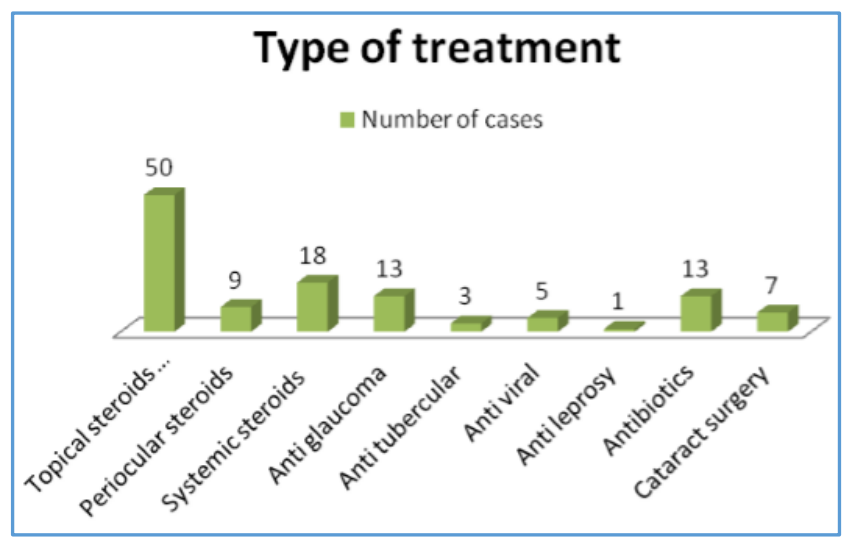

Table 9. Treatment

In the present study, all the 50 patients $(100 \%)$ were treated with topical steroids and cycloplegics-mydriatics. Periocular steroid was given in 9 patients (18\%), of which one had bilateral chronic anterior uveitis and received injections to both the eyes. Systemic steroids were used in 18 patients (36\%) which included 6 patients of phacolytic uveitis, 5 herpetic uveitis patients, 3 patients of TB, 2 idiopathic and one each in leprosy and psoriatic patient; 13 patients (26\%) received antiglaucoma therapy; 3 patients (6\%) received anti-TB drugs, antivirals were considered in 5 cases $(10 \%)$ and all of them had herpetic anterior uveitis. One patient who had already been started on antileprosy therapy was continued. Systemic antibiotics were given in 13 patients $(26 \%)$ (7 underwent cataract extraction, 4 chronic idiopathic cases, one each in inflammatory bowel disease and septic arthritis).

Majority of patients responded well to medical line of treatment. A case of visually significant complicated cataract underwent synechiaeotomy and extracapsular cataract extraction with posterior chamber intraocular lens implantation.

\section{DISCUSSION}

The present study was conducted in the Department of Ophthalmology, Santhiram Medical College, Nandyal during the period of December 2014 to May 2016 and fifty cases of anterior uveitis were studied.

The incidence was found to be high between $20-40$ years of age $(64 \%)$ and less common over sixty years $(10 \%)$. Idiopathic anterior uveitis was the commonest cause, which can be explained by high antigenicity found in this age group. In older age group, anterior uveitis was usually of phacolytic origin.

It was observed that males were affected more (56\%) compared to females (44\%). This may be because men tend to seek medical attention more often than women and socioeconomic habits may put male patients at a greater risk for development of anterior uveitis. In Rathinam et al study, $61.3 \%$ were males and $38.7 \%$ were females. Alezandro Rodriguez et al reported $38.9 \%$ male and $61.1 \%$ female involvement in their study. 


\begin{tabular}{|c|c|c|c|}
\hline & Present & $\begin{array}{c}\text { Rathinam } \\
\text { et al }\end{array}$ & $\begin{array}{c}\text { Alezandro Rodriguez } \\
\text { et al6 }\end{array}$ \\
\hline Males & $56 \%$ & $61.3 \%$ & $38.9 \%$ \\
\hline Females & $44 \%$ & $38.7 \%$ & $61.1 \%$ \\
\hline \multicolumn{3}{|c|}{ Table 10. Gender Comparison } \\
\hline
\end{tabular}

Majority of patients were labourers (46\%). Most common cause of anterior uveitis in labourers was blunt trauma. This may be due to occupational exposure.

Majority of patients came with unilateral presentation (90\%). This finding was comparable with that of Rathinam et al study (85.3\%). However, there was no significant predilection for either the right or left eye.

\begin{tabular}{|c|c|c|}
\hline & Present & Rathinam et al \\
\hline Unilateral & $90 \%$ & $85.3 \%$ \\
\hline Bilateral & $10 \%$ & $14.7 \%$ \\
\hline \multicolumn{2}{|r|}{ Table 11. Laterality Comparison } \\
\hline
\end{tabular}

The most common presentation was acute iridocyclitis (76\%) than chronic (18\%) and recurrent iridocyclitis (6\%). Rathinam et al reported $71.9 \%$ acute, $24.3 \%$ chronic and $3.8 \%$ recurrent. The findings are comparable in both the studies.

\begin{tabular}{|c|c|c|}
\hline & Present & Rathinam et al $^{4}$ \\
\hline Acute & $76 \%$ & $71.9 \%$ \\
\hline Chronic & $18 \%$ & $24.3 \%$ \\
\hline Recurrent & $6 \%$ & $3.8 \%$ \\
\hline Table 12. Chronicity Comparison \\
\hline
\end{tabular}

In this study, 45 patients (90\%) had non-granulomatous inflammation and in 5 patients (10\%) it was granulomatous. Findings are comparable with previous studies. Out of 5 granulomatous inflammation, 4 were chronic and 1 patient had recurrent presentation. Granulomatous type of inflammation was observed in three patients of tuberculosis, one patient of herpes and one patient of leprosy.

\begin{tabular}{|c|c|c|c|}
\hline & Present & $\begin{array}{c}\text { Rathinam } \\
\text { et al }\end{array}$ & $\begin{array}{c}\text { Alezandro } \\
\text { Rodriguez et al6 }\end{array}$ \\
\hline Granulomatous & $10 \%$ & $18.8 \%$ & $12.4 \%$ \\
\hline $\begin{array}{c}\text { Non- } \\
\text { granulomatous }\end{array}$ & $90 \%$ & $81.2 \%$ & $87.6 \%$ \\
\hline \multicolumn{3}{|c|}{ Table 13. Comparison of Type of Inflammation } \\
\hline
\end{tabular}

\begin{tabular}{|c|c|c|c|c|c|}
\hline $\begin{array}{c}\text { Sl. } \\
\text { No. }\end{array}$ & Aetiology & $\begin{array}{c}\text { Present } \\
\text { Study } \\
(\mathbf{n = 5 0 )}\end{array}$ & $\begin{array}{c}\text { Rathinam } \\
\text { et al } \\
(\mathbf{n = 5 0 2 8})\end{array}$ & $\begin{array}{c}\text { Singh } \\
\text { et al } 7 \\
(\mathbf{n = 6 0 7})\end{array}$ & $\begin{array}{c}\text { Henderly } \\
\text { et al } \\
(\mathbf{n}-\mathbf{1 6 7})\end{array}$ \\
\hline 1 & Idiopathic & 42 & 44.6 & 61.3 & 43.52 \\
\hline 2 & Blunt trauma & 20 & 7.7 & - & 2.52 \\
\hline 3 & Phacolytic & 12 & 3.5 & - & - \\
\hline 4 & Herpes zoster & 10 & 8.6 & 1.8 & 8.99 \\
\hline 5 & Tuberculosis & 6 & 4 & 7.9 & - \\
\hline 6 & Septic focus & 2 & - & - & - \\
\hline 7 & $\begin{array}{c}\text { Iridocyclitis } \\
\text { with arthritis }\end{array}$ & 2 & 7.1 & - & - \\
\hline 8 & $\begin{array}{c}\text { Fuchs' } \\
\text { heterochromic } \\
\text { iridocyclitis }\end{array}$ & 2 & 8.4 & 5.1 & 6.47 \\
\hline 9 & \multicolumn{6}{|c|}{ Leprosy } & 2 & 2.1 & 0.8 & - \\
\hline 10 & \multicolumn{6}{|c|}{ IBD } & 2 & - & - & 1.08 \\
\hline \multicolumn{6}{|c|}{ Table 14. Comparison of Aetiological } \\
\hline
\end{tabular}

In the present study, blunt trauma (20\%) was the most common cause of anterior uveitis followed by phacolytic (12\%) aetiology. Although herpes zoster accounted for $10 \%$ of the cases, which is comparable with other two studies where it stood first is not the most common in present study. However, it was the most common infectious cause in our study; $6 \%$ of the patients had tubercular anterior uveitis which is comparable with Rathinam et al and Singh et al study, whereas there is no data in Henderly et al study. This difference may be because all other studies were conducted at referral centres, where cases usually chronic and recurrent ones are referred from primary and secondary centres. Whereas present study was done in a general ophthalmic clinic and most people were from villages.

No complications were seen in 37 eyes (67.27\%). Complications were commonly noted in chronic and recurrent cases. Most common complication observed was persistent posterior synechiae in 13 eyes (23.64\%), cataract in 8 eyes (14.54\%). Secondary glaucoma was seen in 7 eyes (12.73\%), which included 2 herpetic eyes, both the eyes in a psoriatic patient, two idiopathic and one eye in TB anterior uveitis. Iris atrophy was seen in 3 eyes (5.45\%), two of them in a leprosy patient and the third was in a herpetic patient and macular oedema was seen in 1 eye (1.82\%).

A short differential diagnosis was made in each case after complete ocular and systemic examination with tailored approach to the laboratory investigations.

All patients were treated medically by topical steroids and cycloplegics-mydriatics. Treatment with antibiotics, antitubercular drugs, antileprosy and antiviral drugs were considered in appropriate cases. Periocular and systemic steroids were used in cases with severe inflammation, which was not controlled by topical steroids. A case of visually significant complicated cataract underwent synechiaeotomy and extracapsular cataract extraction with posterior chamber intraocular lens implantation. Cataract extraction with posterior chamber intraocular lens implantation was done in all cases of phacolytic anterior uveitis. In all cases, surgery was done under the cover of systemic steroids.

Majority of the patients responded well to the medical line of treatment.

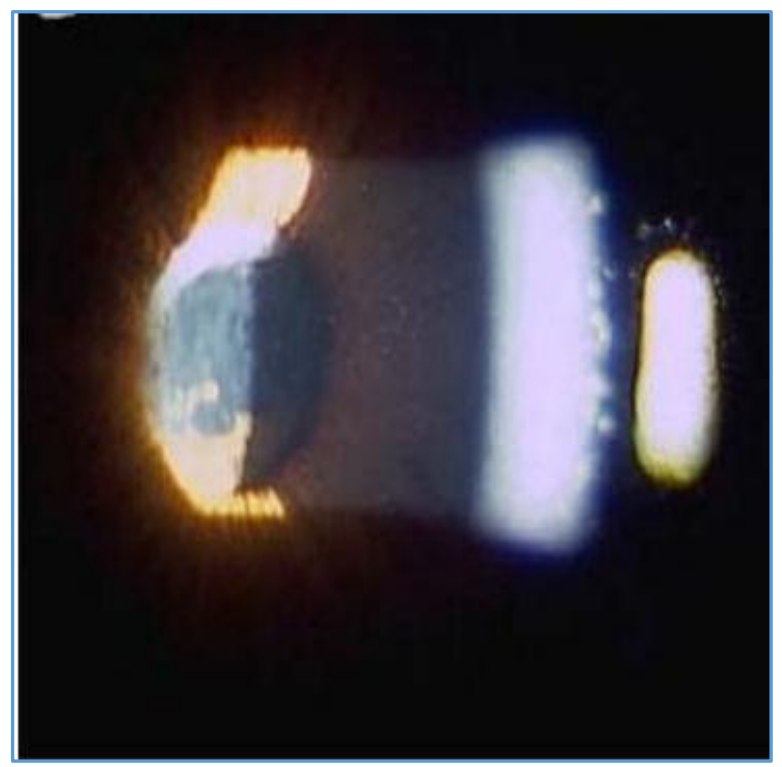

Cells and Flare +3 in AC 3x $1 \mathrm{~mm}$ Beam 


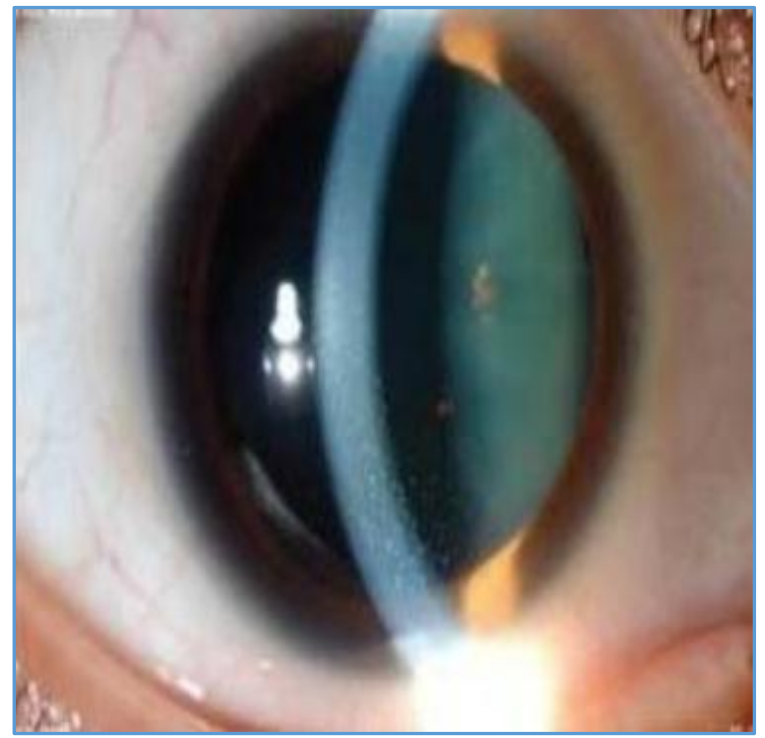

Fine KP's

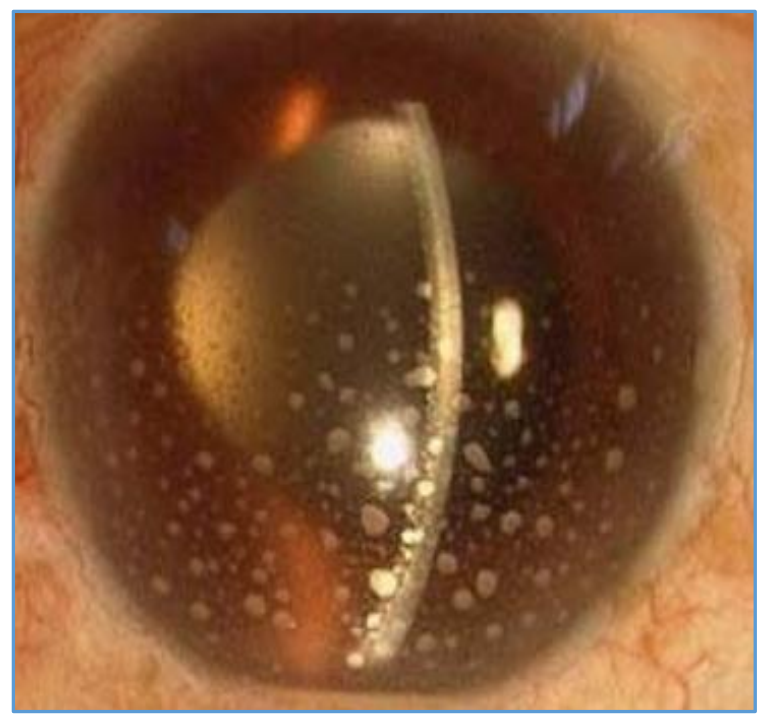

Mutton-Fat KP's

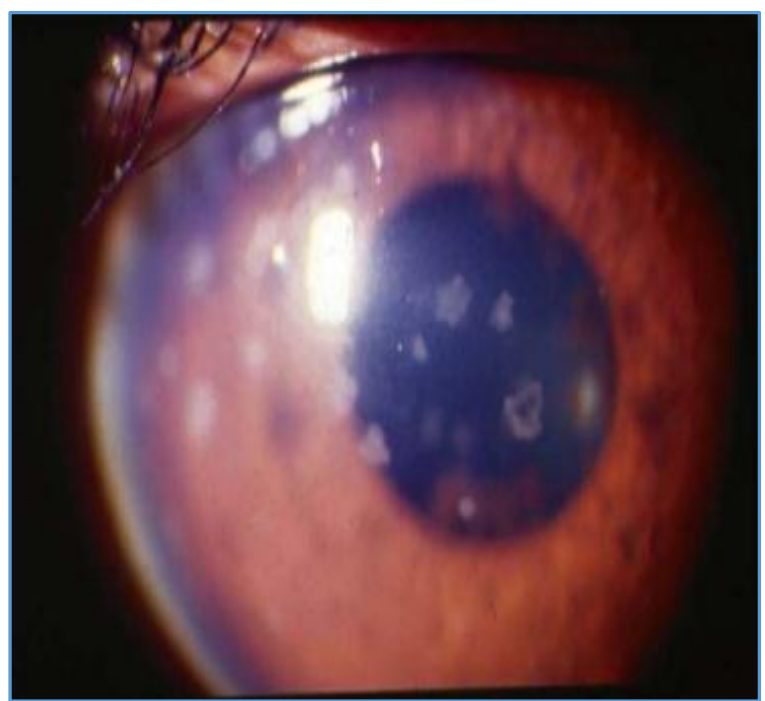

Large Old KP's

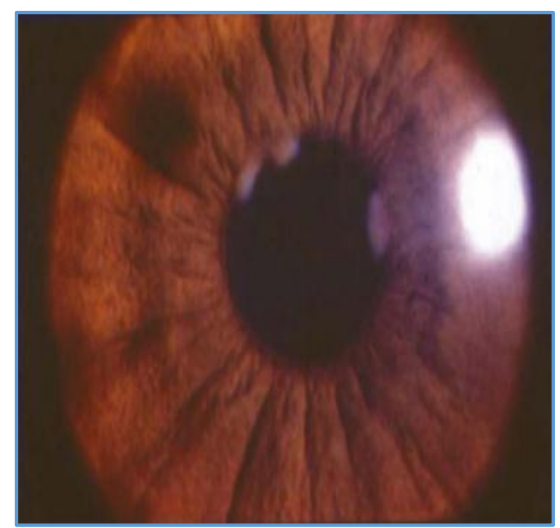

Koeppe Nodules

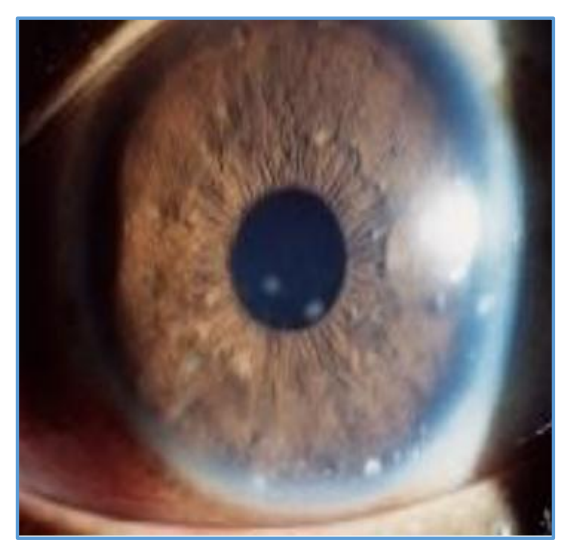

Busacca Nodules with few Mutton-Fat KP's

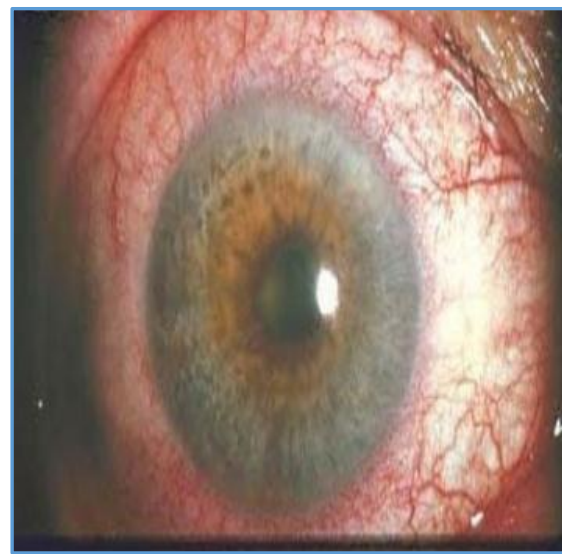

Iris Atrophy

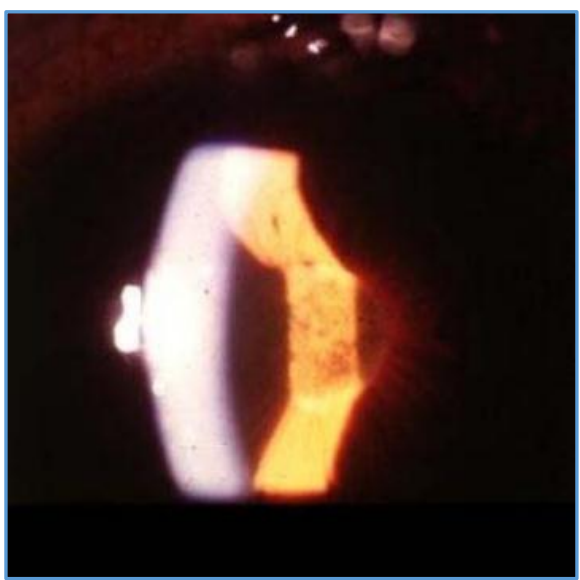

Iris Bombe with Neovascularisation 


\begin{tabular}{|c|c|c|c|c|c|c|c|c|c|c|c|c|c|c|c|c|c|}
\hline \multirow[t]{3}{*}{$\frac{\dot{z}}{\dot{m}}$} & \multirow[t]{3}{*}{ 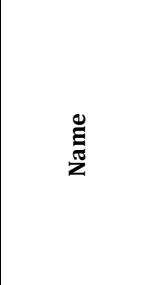 } & \multirow[t]{3}{*}{ 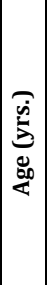 } & \multirow[t]{3}{*}{ 麃 } & \multirow[t]{3}{*}{$\begin{array}{l}\dot{0} \\
z \\
o \\
ٍ\end{array}$} & \multirow{3}{*}{ 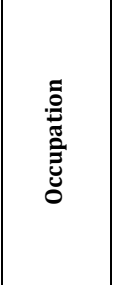 } & \multirow[t]{3}{*}{ ப் } & \multirow[t]{3}{*}{ స̂े } & \multirow{3}{*}{ 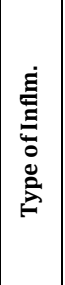 } & \multirow[t]{3}{*}{$\begin{array}{l}\frac{\overrightarrow{0}}{00} \\
\frac{0}{0} \\
\frac{0}{4} \\
\frac{0}{4}\end{array}$} & \multirow[t]{3}{*}{ 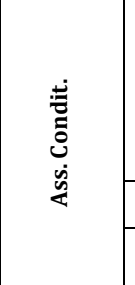 } & \multicolumn{4}{|c|}{ 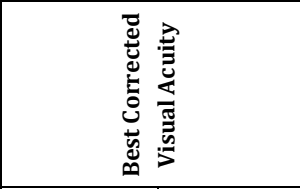 } & \multirow[t]{3}{*}{ 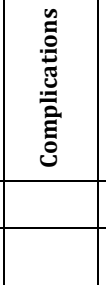 } & \multirow[t]{3}{*}{ 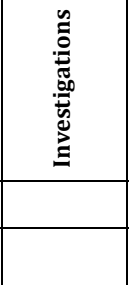 } & \multirow[t]{3}{*}{ 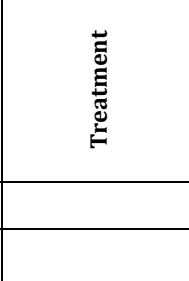 } \\
\hline & & & & & & & & & & & \multicolumn{2}{|c|}{ 똟 } & \multicolumn{2}{|c|}{ 떡 } & & & \\
\hline & & & & & & & & & & & ติ & $\varepsilon$ & 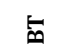 & \& & & & \\
\hline 1 & Sumana & 28 & $\mathrm{~F}$ & 68004 & Official & $\mathrm{RE}$ & Acute & $\mathrm{NG}$ & Idiopathic & & $6 / 9$ & $6 / 6$ & $6 / 6$ & $6 / 6$ & & & $\mathrm{TS}+\mathrm{CM}$ \\
\hline 2 & Basappa & 54 & $\mathrm{M}$ & 6863 & Labourer & $\mathrm{RE}$ & Chronic & G & TB & \begin{tabular}{|c|} 
Pulmonary \\
TB, DM
\end{tabular} & $6 / 60$ & $6 / 12$ & $6 / 6$ & $6 / 6$ & \begin{tabular}{|c|} 
PPS, Sec. \\
glu
\end{tabular} & $\begin{array}{c}\text { Chest } \mathrm{x}^{-} \\
\text {ray+ }\end{array}$ & $\begin{array}{c}\mathrm{TS}+\mathrm{CM}+\mathrm{PS}+\mathrm{SS}+ \\
\mathrm{AG}+\text { antiTB }\end{array}$ \\
\hline 3 & Munaff & 36 & $\mathrm{M}$ & 77903 & Official & LE & Acute & NG & Idiopathic & & $6 / 6$ & $6 / 6$ & $6 / 36$ & $6 / 9$ & & & $\mathrm{TS}+\mathrm{CM}$ \\
\hline 4 & Kamakshi & 22 & $\mathrm{~F}$ & 126681 & Labourer & LE & Acute & $\mathrm{NG}$ & Idiopathic & & $6 / 6$ & $6 / 6$ & $6 / 12$ & $6 / 6$ & & & $\mathrm{TS}+\mathrm{CM}$ \\
\hline 5 & Shankarappa & 64 & M & 82005 & Business & $\mathrm{BE}$ & Chronic & G & TB & \begin{tabular}{|c|} 
Pulmonary \\
TB
\end{tabular} & $\mathrm{CF} 2 \mathrm{~m}$ & $6 / 36$ & CF1m & $6 / 36$ & \begin{tabular}{|c|} 
PPS \& \\
PSC, BE \\
\end{tabular} & $\begin{array}{c}\text { Chest } x^{-} \\
\text {ray+ }\end{array}$ & $\begin{array}{c}\mathrm{TS}+\mathrm{CM}+\mathrm{SS}+\text { anti } \\
\mathrm{TB}+\mathrm{SA}+\mathrm{ECCE}(\mathrm{BE})\end{array}$ \\
\hline 6 & Mohamad & 42 & $\mathrm{M}$ & 54936 & Official & $\mathrm{RE}$ & Acute & $\mathrm{NG}$ & Trauma & & $6 / 36$ & $6 / 6$ & $6 / 6$ & $6 / 6$ & & & $\mathrm{TS}+\mathrm{CM}$ \\
\hline 7 & Ramani & 25 & $\mathrm{~F}$ & 155721 & Labourer & LE & Acute & $\mathrm{NG}$ & Trauma & & $6 / 6$ & $6 / 6$ & $6 / 12$ & $6 / 6$ & & & $\mathrm{TS}+\mathrm{CM}$ \\
\hline 8 & Muniyamma & 35 & $\mathrm{~F}$ & 116373 & $\begin{array}{c}\text { House } \\
\text { wife }\end{array}$ & $\mathrm{RE}$ & Acute & $\mathrm{NG}$ & Idiopathic & Myopia & $6 / 36$ & $6 / 9$ & $6 / 6$ & $6 / 6$ & & & $\mathrm{TS}+\mathrm{CM}$ \\
\hline 9 & Daruka & 29 & M & 44885 & Labourer & $\mathrm{RE}$ & Acute & $\mathrm{NG}$ & Herpes zoster & $\begin{array}{c}\text { Skin } \\
\text { lesions }\end{array}$ & $6 / 12$ & $6 / 6$ & $6 / 6$ & $6 / 6$ & & \begin{tabular}{|c|} 
Clinical \\
Diagnosis \\
\end{tabular} & $\begin{array}{c}\text { TS+CM+SS+ } \\
\text { Antiviral }\end{array}$ \\
\hline 10 & Panchakshari & 27 & $\mathrm{M}$ & 10571 & \begin{tabular}{|l|} 
Labourer \\
\end{tabular} & LE & Acute & $\mathrm{NG}$ & Trauma & & $6 / 6$ & $6 / 6$ & $6 / 18$ & $6 / 6$ & & & $\mathrm{TS}+\mathrm{CM}$ \\
\hline 11 & Nagarathna & 33 & $\mathrm{~F}$ & 93718 & $\begin{array}{c}\text { House } \\
\text { wife }\end{array}$ & $\mathrm{RE}$ & Acute & $\mathrm{NG}$ & Idiopathic & & $6 / 18$ & $6 / 9$ & $6 / 6$ & $6 / 6$ & & & $\mathrm{TS}+\mathrm{CM}$ \\
\hline 12 & Rudreshi & 34 & $\mathrm{M}$ & 38592 & Labourer & $\mathrm{RE}$ & Acute & $\mathrm{NG}$ & Herpes zoster & $\begin{array}{c}\text { Skin } \\
\text { lesions }\end{array}$ & $6 / 24$ & $6 / 9$ & $6 / 6$ & $6 / 6$ & IA & $\begin{array}{c}\text { Clinical } \\
\text { Diagnosis }\end{array}$ & $\begin{array}{c}\mathrm{TS}+\mathrm{CM}+\mathrm{SS}+ \\
\text { antiviral }\end{array}$ \\
\hline 13 & Sundharamma & 30 & $\mathrm{~F}$ & 41321 & Official & $\mathrm{BE}$ & Recurrent & $\mathrm{NG}$ & IAA & $\begin{array}{l}\text { Psoriatic } \\
\text { arthritis }\end{array}$ & $6 / 24$ & $6 / 18$ & $6 / 60$ & $6 / 18$ & \begin{tabular}{|c|} 
Sec. glu \\
BE \\
\end{tabular} & HLA-B27+ & $\begin{array}{c}\mathrm{TS}+\mathrm{CM}+\mathrm{PS} \\
(\mathrm{LE})+\mathrm{SS}+\mathrm{AG} \\
\end{array}$ \\
\hline 14 & Papanna & 65 & M & 26059 & Labourer & LE & Acute & $\mathrm{NG}$ & Phacolytic & $\mid \begin{array}{c}\text { SIMC (RE) } \\
\text { HTN }\end{array}$ & $6 / 36$ & $6 / 36$ & $\begin{array}{l}\mathrm{PL}+ \\
\mathrm{PR}+\end{array}$ & $6 / 12$ & & & $\begin{array}{l}\mathrm{TS}+\mathrm{CM}+\mathrm{SS}+\mathrm{AG}+ \\
\mathrm{SA}+\mathrm{ECCE}, \mathrm{PCIOL} \\
\text { (LE) }\end{array}$ \\
\hline 15 & Jagadeesh & 31 & $\mathrm{M}$ & 116772 & Labourer & LE & cute & $\mathrm{NG}$ & Idiopathic & & $6 / 6$ & $6 / 6$ & $5 / 12$ & $6 / 6$ & & & $\mathrm{TS}+\mathrm{CM}$ \\
\hline 16 & Govinda & 20 & $\mathrm{M}$ & 119217 & Student & $\mathrm{RE}$ & cute & $\mathrm{NG}$ & Trauma & & $6 / 9$ & $6 / 6$ & $6 / 6$ & $6 / 6$ & & & $\mathrm{TS}+\mathrm{CM}$ \\
\hline 17 & Gouramma & 57 & $\mathrm{~F}$ & 19261 & $\begin{array}{l}\text { House } \\
\text { wife }\end{array}$ & $\mathrm{RE}$ & Acute & $\mathrm{NG}$ & Phacolytic & PSC (LE) & $\mathrm{HM}+$ & $6 / 9$ & $6 / 12$ & $6 / 12$ & & & $\begin{array}{c}\mathrm{TS}+\mathrm{CM}+\mathrm{SS}+\mathrm{AG}+ \\
\mathrm{SA}+\mathrm{SICS}, \mathrm{PCIOL} \\
(\mathrm{RE})\end{array}$ \\
\hline 18 & Jayanthi & 45 & $\mathrm{~F}$ & 13734 & $\begin{array}{l}\text { House } \\
\text { wife }\end{array}$ & $\mathrm{BE}$ & Chronic & $\mathrm{NG}$ & Idiopathic & & $\mathrm{CF} 2 \mathrm{~m}$ & $6 / 9$ & $6 / 60$ & $6 / 24$ & $\begin{array}{c}\text { PPS \& } \\
\text { PSC, BE }\end{array}$ & & $\begin{array}{c}\mathrm{TS}+\mathrm{CM}+\mathrm{PS}(\mathrm{BE})+\mathrm{S} \\
\mathrm{S}+\mathrm{AG}+\mathrm{SA}+\mathrm{ECCE} \\
(\mathrm{RE})\end{array}$ \\
\hline 19 & Satywathi & 40 & $\mathrm{~F}$ & \begin{tabular}{|l|}
23498 \\
\end{tabular} & Official & $\mathrm{RE}$ & Acute & $\mathrm{NG}$ & diopathic & & $6 / 12$ & $6 / 6$ & $6 / 6$ & $6 / 6$ & & & $\mathrm{TS}+\mathrm{CM}$ \\
\hline 20 & Yogesh & 23 & $\mathrm{M}$ & 129049 & Labourer & $\mathrm{RE}$ & Acute & $\mathrm{NG}$ & Idiopathic & & $6 / 24$ & $6 / 9$ & $6 / 6$ & $6 / 6$ & & & $\mathrm{TS}+\mathrm{CM}$ \\
\hline 21 & $\begin{array}{c}\text { Meenakshamm } \\
\text { a }\end{array}$ & 72 & $\mathrm{~F}$ & 34491 & $\begin{array}{c}\text { House } \\
\text { wife }\end{array}$ & LE & Acute & $N G$ & Phacolytic & \begin{tabular}{|c|} 
SIMC (RE), \\
DM
\end{tabular} & $6 / 60$ & $6 / 36$ & $\begin{array}{l}\mathrm{PL}+ \\
\mathrm{PR}+\end{array}$ & $6 / 9$ & & & $\begin{array}{l}\mathrm{TS}+\mathrm{CM}+\mathrm{SS}+\mathrm{AG}+\mathrm{SA} \\
+\mathrm{SICS}, \mathrm{PCIOL}(\mathrm{LE})\end{array}$ \\
\hline 22 & Prahalhad & 44 & $\mathrm{M}$ & 25420 & Official & LE & Acute & NG & Idiopathic & & $6 / 6$ & $6 / 6$ & $6 / 9$ & $6 / 6$ & & & $\mathrm{TS}+\mathrm{CM}$ \\
\hline 23 & Zarrena & 24 & \begin{tabular}{|l|}
$\mathrm{F}$ \\
\end{tabular} & \begin{tabular}{|l|}
96437 \\
\end{tabular} & \begin{tabular}{|l|} 
Labourer \\
\end{tabular} & $\mathrm{RE}$ & Acute & $\mathrm{NG}$ & IBD & & $6 / 12$ & $6 / 6$ & $6 / 6$ & $6 / 6$ & & \begin{tabular}{|l|} 
HLA-B27+ \\
\end{tabular} & $\mathrm{TS}+\mathrm{CM}+\mathrm{SA}$ \\
\hline 24 & Shanthakumar & 25 & $\mathrm{M}$ & 11417 & Labourer & $\mathrm{RE}$ & Acute & $\mathrm{NG}$ & Trauma & & $6 / 18$ & $6 / 6$ & $6 / 6$ & $6 / 6$ & & & $\mathrm{TS}+\mathrm{CM}$ \\
\hline 25 & Laksmana & 37 & $\mathrm{M}$ & 27892 & Labourer & $\mathrm{RE}$ & Chronic & $N G$ & Herpes zoster & & $6 / 60$ & $6 / 12$ & $6 / 6$ & $6 / 6$ & \begin{tabular}{|c|}
$\begin{array}{c}\text { PPS, Sec } \\
\text { glu }\end{array}$ \\
\end{tabular} & & $\begin{array}{c}\mathrm{TS}+\mathrm{CM}+\mathrm{SS}+\mathrm{AG}+ \\
\text { Antiviral }\end{array}$ \\
\hline 26 & Bhaskar & 50 & $\mathrm{M}$ & 49371 & Official & $\mathrm{RE}$ & Chronic & $\mathrm{NG}$ & Septic foci & $\begin{array}{c}\text { Arthritis R } \\
\text { knee, DM }\end{array}$ & $6 / 24$ & $6 / 9$ & $6 / 6$ & $6 / 6$ & & $\begin{array}{c}\text { Synovial } \\
\text { fluid } \mathrm{C} / \mathrm{S}+\end{array}$ & $\mathrm{TS}+\mathrm{CM}+\mathrm{PS}+\mathrm{SA}$ \\
\hline 27 & Jayashree & 21 & $\mathrm{~F}$ & 26381 & udent & LE & cute & NG & Idiopathic & & $6 / 6$ & $6 / 6$ & $6 / 6$ & $6 / 6$ & & & $\mathrm{TS}+\mathrm{CM}$ \\
\hline 28 & Girijamma & 46 & $\mathrm{~F}$ & 92168 & Labourer & $\mathrm{BE}$ & Recurrent & G & TB & $\mid \begin{array}{c}\text { Pulmonary } \\
\text { TB }\end{array}$ & $6 / 18$ & $6 / 12$ & $6 / 60$ & $6 / 36$ & \begin{tabular}{|c|} 
PPS BE \\
$\&$ PSC \\
(LE) \\
\end{tabular} & $\begin{array}{l}\text { Chest } x^{-} \\
\text {ray+ }\end{array}$ & $\begin{array}{c}\mathrm{TS}+\mathrm{CM}+\mathrm{PS}(\mathrm{LE})+ \\
\mathrm{SS}+\text { antiTB }\end{array}$ \\
\hline 29 & Shanthamma & 55 & $\mathrm{~F}$ & 32229 & $\begin{array}{l}\text { House } \\
\text { wife }\end{array}$ & $\mathrm{RE}$ & Acute & $\mathrm{NG}$ & Phacolytic & \begin{tabular}{|} 
PSC (LE), \\
DM
\end{tabular} & $\begin{array}{c}\mathrm{CF} 1 / \\
2 \mathrm{~m}\end{array}$ & $6 / 9$ & $6 / 24$ & $6 / 24$ & & & $\begin{array}{c}\mathrm{TS}+\mathrm{CM}+\mathrm{SS}+\mathrm{AG}+\mathrm{SA} \\
+\mathrm{ECCE}, \mathrm{PCIOL} \\
\text { (RE) }\end{array}$ \\
\hline 30 & Barkat Ali & 30 & $\mathrm{M}$ & 87639 & Business & LE & Recurrent & $\mathrm{NG}$ & Idiopathic & & $6 / 6$ & $6 / 6$ & $6 / 36$ & $6 / 9$ & Sec. glu & & $\begin{array}{c}\mathrm{TS}+\mathrm{CM}+\mathrm{PS}+ \\
\mathrm{SS}+\mathrm{AG}+\mathrm{SA}\end{array}$ \\
\hline 31 & iden & 26 & $\mathrm{M}$ & \begin{tabular}{|l|l}
39452 \\
\end{tabular} & \begin{tabular}{|l|} 
Labourer \\
\end{tabular} & $\mathrm{RE}$ & cute & $\mathrm{NG}$ & rauma & & $6 / 12$ & $6 / 6$ & $6 / 6$ & $6 / 6$ & & & $\mathrm{TS}+\mathrm{CM}$ \\
\hline 32 & Pankaja & 38 & $\mathrm{~F}$ & 2894 & Official & LE & Chronic & $N G$ & Idiopathic & & $6 / 6$ & $6 / 6$ & $6 / 60$ & $6 / 18$ & $\begin{array}{c}\text { PPS, PSC } \\
\text { (LE) }\end{array}$ & Yield less & $\mathrm{TS}+\mathrm{CM}+\mathrm{PS}+\mathrm{SA}$ \\
\hline 33 & Manjunath & 48 & $\mathrm{M}$ & 40563 & Official & $\mathrm{RE}$ & Acute & $N G$ & Herpes zoster & Skin lesions & $6 / 18$ & $6 / 6$ & $6 / 6$ & $6 / 6$ & & $\begin{array}{c}\text { Clinical } \\
\text { Diagnosis }\end{array}$ & $\begin{array}{c}\text { TS+CM+SS+ } \\
\text { Antiviral }\end{array}$ \\
\hline 34 & Sharanappa & 22 & $\mathrm{M}$ & \begin{tabular}{|l|l|}
29748 \\
\end{tabular} & \begin{tabular}{|l|} 
Labourer \\
\end{tabular} & LE & ute & NG & auma & & $6 / 6$ & $6 / 6$ & $/ 9$ & $6 / 6$ & & & $\mathrm{TS}+\mathrm{CM}$ \\
\hline 35 & Susheel & 58 & $\mathrm{~F}$ & 206934 & $\begin{array}{c}\text { House } \\
\text { wife }\end{array}$ & $\mathrm{RE}$ & ate & $N G$ & colytic & SIMC (LE) & $\begin{array}{l}\mathrm{PL}+ \\
\mathrm{PR}+\end{array}$ & 9 & $6 / 60$ & $6 / 60$ & & & $\begin{array}{l}\text { TS+CM+SS+AG+SA } \\
+ \text { SICS,PCIOL (RE) }\end{array}$ \\
\hline 36 & \begin{tabular}{|c|}
$\begin{array}{c}\text { Venkatalaksh } \\
\mathrm{mi}\end{array}$ \\
\end{tabular} & 40 & $\mathrm{~F}$ & 46253 & $\begin{array}{l}\text { House } \\
\text { wife }\end{array}$ & $\mathrm{RE}$ & Acute & $\mathrm{NG}$ & Idiopathic & & $6 / 36$ & $6 / 12$ & $6 / 6$ & $6 / 6$ & $\mathrm{ME}$ & & $\begin{array}{c}\text { TS+CM+PS+NSAID } \\
\text { S }\end{array}$ \\
\hline 37 & Giriyappa & 26 & $\mathrm{M}$ & 50062 & Labourer & $\mathrm{RE}$ & Acute & $\mathrm{NG}$ & Idiopathic & & $6 / 12$ & $6 / 6$ & $6 / 6$ & $6 / 6$ & & & $\mathrm{TS}+\mathrm{CM}$ \\
\hline 38 & Chandrappa & 62 & $\mathrm{M}$ & 61248 & Business & $\mathrm{RE}$ & Acute & $\mathrm{NG}$ & Phacolytic & \begin{tabular}{|c|} 
SIMC (LE), \\
HTN
\end{tabular} & $\begin{array}{l}\mathrm{PL}+ \\
\mathrm{PR}+\end{array}$ & $6 / 18$ & $6 / 24$ & $6 / 24$ & & & $\begin{array}{l}\text { TS+CM+SS+AG+SA } \\
+ \text { SICS,PCIOL (RE) }\end{array}$ \\
\hline
\end{tabular}




\begin{tabular}{|c|c|c|c|c|c|c|c|c|c|c|c|c|c|c|c|c|c|}
\hline 39 & Ambuja & 43 & $\mathrm{~F}$ & 44380 & Labourer & LE & Chronic & G & Herpes zoster & HIV & $6 / 6$ & $6 / 6$ & $6 / 60$ & $6 / 12$ & \begin{tabular}{|c} 
PPS, Sec. \\
glu
\end{tabular} & \begin{tabular}{|c|} 
Clinical \\
Diagnosis \\
\end{tabular} & $\begin{array}{l}\mathrm{TS}+\mathrm{CM}+\mathrm{SS}+ \\
\mathrm{AG}+\text { antiviral }\end{array}$ \\
\hline 40 & Jaganath & 28 & $\mathrm{M}$ & 55486 & Labourer & LE & Acute & NG & Trauma & & $6 / 6$ & $6 / 6$ & $6 / 12$ & $6 / 6$ & & & $\mathrm{TS}+\mathrm{CM}$ \\
\hline 41 & Rangamma & 37 & $\mathrm{~F}$ & 114559 & Labourer & LE & Acute & $\mathrm{NG}$ & Idiopathic & & $6 / 6$ & $6 / 6$ & $6 / 9$ & $6 / 6$ & & & $\mathrm{TS}+\mathrm{CM}$ \\
\hline 42 & \begin{tabular}{|} 
Shambhulinga \\
ppa
\end{tabular} & 75 & M & 87542 & Labourer & $\mathrm{BE}$ & Chronic & G & Leprosy & Leprosy & $6 / 60$ & $6 / 36$ & $6 / 60$ & $6 / 24$ & \begin{tabular}{|c|} 
PPS,IA\& \\
PSC BE
\end{tabular} & $\begin{array}{c}\text { Confirmed } \\
\text { case }\end{array}$ & \begin{tabular}{|c|}
$\mathrm{TS}+\mathrm{CM}+\mathrm{SS}+$ anti- \\
Leprosy
\end{tabular} \\
\hline 43 & Kareemsab & 24 & M & 59716 & Labourer & LE & Acute & NG & Idiopathic & & $6 / 6$ & $6 / 6$ & $6 / 18$ & $6 / 6$ & & & $\mathrm{TS}+\mathrm{CM}$ \\
\hline 44 & Suresh & 27 & $\mathrm{M}$ & 24369 & Official & $\mathrm{RE}$ & Acute & NG & Idiopathic & & $6 / 12$ & $6 / 6$ & $6 / 6$ & $6 / 6$ & & & $\mathrm{TS}+\mathrm{CM}$ \\
\hline 45 & Lingamma & 45 & $\mathrm{~F}$ & 61748 & $\begin{array}{l}\text { House } \\
\text { wife }\end{array}$ & $\mathrm{RE}$ & Acute & NG & FHI & & $6 / 9$ & $6 / 6$ & $6 / 6$ & $6 / 6$ & & & $\mathrm{TS}+\mathrm{CM}$ \\
\hline 46 & Nagamma & 39 & $\mathrm{~F}$ & 84250 & Labourer & LE & Acute & $\mathrm{NG}$ & Trauma & & $6 / 6$ & $6 / 6$ & $6 / 24$ & $6 / 9$ & & & $\mathrm{TS}+\mathrm{CM}$ \\
\hline 47 & Nijaguna & 23 & $\mathrm{M}$ & 21447 & Labourer & LE & Acute & NG & Idiopathic & & $6 / 6$ & $6 / 6$ & $6 / 18$ & $6 / 6$ & & & $\mathrm{TS}+\mathrm{CM}$ \\
\hline 48 & Krishnaveni & 60 & $\mathrm{~F}$ & 413 & $\begin{array}{l}\text { House } \\
\text { wife }\end{array}$ & $\mathrm{RE}$ & Chronic & NG & Idiopathic & $\begin{array}{l}\text { PSC (LE), } \\
\text { DM }\end{array}$ & CF4m & $6 / 60$ & $6 / 24$ & $6 / 24$ & $\begin{array}{c}\text { PPS, Sec. } \\
\text { glu }\end{array}$ & & $\mathrm{TS}+\mathrm{CM}+\mathrm{PS}+\mathrm{AG}+\mathrm{SA}$ \\
\hline 49 & Niaz noorulla & 32 & $\mathrm{M}$ & 89273 & Official & LE & Acute & NG & Idiopathic & & $6 / 6$ & $6 / 6$ & $6 / 24$ & $6 / 6$ & & & $\mathrm{TS}+\mathrm{CM}$ \\
\hline 50 & Channabasava & 21 & $\mathrm{M}$ & 98261 & Labourer & LE & Acute & NG & Trauma & & $6 / 6$ & $6 / 6$ & $6 / 12$ & $6 / 6$ & & & $\mathrm{TS}+\mathrm{CM}$ \\
\hline
\end{tabular}

\section{CONCLUSION}

This is a prospective study done during December 2014 to May 2016. We studied 50 cases of anterior uveitis with emphasis on evaluating the possible aetiology, associated complications and treatment outcome.

1. Anterior uveitis is a relatively common ocular condition.

2. In the present study, males were affected more than the females.

3. Patients in the age group of 20 - 40 years were commonly involved.

4. Labourers were by far the commonly involved group in this study; blunt trauma was the leading cause in them and this may be due the risk of injury at their work place. Next common group was that of officials and most of them had idiopathic disease.

5. Majority of the patients had acute presentation.

6. $90 \%$ of the patients presented with unilateral ocular involvement, among them right eye involvement was slightly more than the left eye involvement in the ratio of 5:4. Both eye involvement was seen in $10 \%$ of the patients, all of them had either chronic or recurrent disease and four of them had identifiable aetiology.

7. Nongranulomatous inflammation was the commonest form accounting for $90 \%$ of the cases. Granulomatous inflammation was seen only in chronic cases excepting one patient who had recurrent anterior uveitis.

8. Despite of efforts, diagnosis remained obscure in $42 \%$ of the cases, blunt trauma was the most common identifiable cause in $20 \%$ of the cases, more so in labourers. Herpetic aetiology topped the list of infectious cause followed by tuberculosis. Septic foci, iridocyclitis associated with arthritis, Fuchs'.

9. Heterochromic iridocyclitis, leprosy, inflammatory bowel disease are all relatively less common causes of anterior uveitis.

10. The challenge in anterior uveitis is to develop tailored laboratory investigations that will facilitate a diagnosis. This can be done by first considering the probable diagnosis based on the patient profile and then performing tailored laboratory evaluation.

11. A thorough systemic examination should be done to rule out any systemic disease, as it may be an early manifestation of systemic disease.

12. Majority of anterior uveitis patients respond to medical line of treatment.
13. Chronicity increases the risk of complications as does delay in receiving appropriate therapy, but early recognition and treatment of patients who are prone to recurrences can improve their outcome.

14. Early diagnosis and treatment of patients results in good visual prognosis and is the key in management of anterior uveitis.

\section{Summary}

- A clinical prospective study of 50 patients clinically presenting with anterior uveitis was done.

- All patients underwent a thorough systemic and ocular examination.

- Tailored laboratory investigations were done in each case to facilitate diagnosis.

- $\quad$ Patients were put on treatment and were followed up at regular intervals over a period of 6 months.

- Aetiology remained undetermined in most cases. Most common cause in present study was blunt trauma followed by phacolytic anterior uveitis.

- Most common complications were persistent, posterior synechiae and complicated cataract.

- Majority of patients responded well to treatment.

- $70.91 \%$ of the patients regained visual acuity of $6 / 9$ or better.

\section{REFERENCES}

[1] Rathinam SR. CME series. All India ophthalmological society. Uveitis made simple work up and management. 2010.

[2] Sugar J. Stromal corneal dystrophies and ectasias. In: Yanoff M, Duker JS, Augsburger JJ, (eds). Ophthalmology. 2. St. Louis, MO: Mosby 2004:442-4.

[3] Alio J, Ben Ezra D. Priority features of intraocular inflammation. Highlights of Ophthalmology 2002;30(3):1-2.

[4] Rathinam SR, Namperumalsamy P. Global variation and pattern changes in epidemiology of uveitis. Indian J Ophthalmol 2007;55(3):173-83.

[5] Martin TM, Smith JR, Rosenbaum JT. Anterior uveitis: current concepts of pathogenesis and interactions with the spondyloarthropathies. Curr Opin Rheumatol 2002;14(4):337-41. 


\section{Jemds.com}

[6] Rodriguez A, Calonge M, Pedroza-Seres $M$, et al. Referral patterns of uveitis in a tertiary care center. Arch Ophthalmol 1996;114(5):593-9.

[7] Singh R, Gupta V, Gupta A. Pattern of uveitis in a referral eye clinic in north India. Indian J Ophthalmol 2004;52(2):121-5.

\section{Original Research Article}

[8] Henderly DE, Genstler AJ, Smith RE, et al. Changing patterns of uveitis. Am J Ophthalmol 1987;103(2):1316. 